\title{
POLAR SNAKES: A FAST AND ROBUST PARAMETRIC ACTIVE CONTOUR MODEL
}

\author{
Christophe Collewet \\ INRIA Rennes - Bretagne Atlantique, IRISA, Lagadic team, Rennes, France.
}

\begin{abstract}
We present in this paper a way to perform a fast and robust image segmentation and to track a contour along a sequence of images. Our approach is based on a dynamic deformable model. More precisely, we revisit the physics basedmodel proposed in [1] to show the benefit of using a polar description to model the contour, in particular to cope with the well-known initialization problem. Indeed, we show that this way to proceed leads to diagonal and constant matrices in the equations of the snake evolution yielding therefore to a faster algorithm. Experimental results on image segmentation and contour tracking validate the efficiency of this new formulation.
\end{abstract}

Index Terms - Active contour model, polar description, segmentation, contour tracking.

\section{INTRODUCTION}

Since their first introduction in [2], active contours or snakes have proven to be very powerful in various contexts, including image segmentation [3], edge detection [4,5] and target tracking $[6,7]$. An active contour is a deformable curve defined in the image domain that evolves according to internal forces coming from the curve itself and external forces generated from the image (most often gradient-based or regionbased information). Numerous approaches are reported in the literature. Nevertheless, they can be classified into two main categories: geometric snakes $[4,8]$ where the curves is described from a level set representation, or parametric snakes where the curve is either described as a discrete collection of points $[2,9]$ or by an appropriate parametric function such as superquadrics [1] or B-splines [3,6] for example. In this paper we are interested in the latter case, that is in parametric active contours. Indeed, we will consider here robotics issues such as $3 \mathrm{D}$ reconstruction by a moving camera or robot control by visual servoing [10]. In those cases, a high acquisition rate is required and thus the image processing duration has to be as near as possible to the video rate. Currently, it is well known that geometric approaches are too much time consuming and consequently, they cannot be used in this context. In addition, compared to traditional methods based on points, parametric snakes based on parametric functions are less time consuming. Such works are reported for example in [1,5-7].
However, the major drawback of parametric active contours is that they are very sensitive to the initial position of the contour. Indeed, it has to be chosen close to the true contour to ensure its convergence to the correct position. Numerous approaches have been proposed to cope with this problem. A pressure force acts as a force that can inflate or deflate the contour according to the normal at each point belonging to it [11]. Another approach is to introduce an external force based on a distance map between a point belonging to the active contour and the closest boundary point [12]. Multiresolution methods have also been proposed to cope with this problem $[5,13]$. A new external force, called GVF, computed as a diffusion of the image gradient map has been presented in [9] to ensure a convergence even from an initial contour far from the true one. However, computing such forces is very time consuming.

The main contribution of this paper is to show the benefit of using a polar description as the parametric curve to describe the contour. In addition, we introduce an external force derived from an energy term based on the area inside the contour. It is very closed in mind to the pressure forces [11]. However, since the polar description is based on orthogonal functions, the evolution equations (including the energy term) of this kind of active contour are very simple and, consequently, make the computations faster.

This paper is structured as follow. First, we revisit in Section 2 the evolution equations of a physics-based deformable model as proposed in [1] for a generic family functions. Next, this result is used in Section 3 to show that a polar description highly simplifies these equations. Section 4 points out that it becomes very simple to introduce an area-based energy term. The experimental results are presented in Section 5.

\section{EVOLUTION EQUATIONS OF THE CONTOUR}

First, let us consider the generic family curve $\mathcal{C}_{\mathbf{q}}$ depending on a $n$-dimensional parameter vector $\mathbf{q}$ and defined in the image plane by

$$
\mathcal{C}_{\mathbf{q}}(u): \mathbf{x}(u)=\mathbf{x}_{c}+\sum_{l=0}^{l=n} q_{l} \boldsymbol{\Phi}_{l}(u)
$$

$\mathbf{x}_{c}$ being a point inside the contour, $u \in[0,2 \pi]$ and $\boldsymbol{\Phi}_{l}(l=$ $0 \cdots n$ ) some $2 \mathrm{~d}$ vector functions. Note that $\mathcal{C}_{\mathbf{q}}$ writes as a 
linear form with respect to $q$.

To derive the evolution equation of $\mathcal{C}_{\mathbf{q}}$, we search the evolution of $\mathbf{q}$ as proposed in [1]. More precisely, this curve is supposed to be embedded in a viscous medium and supposed to be undergone to internal forces $\mathbf{f}_{i}$ and to external forces $\mathbf{f}_{e}$. This problem can be set according to the formalism of Lagrangian mechanics when considering $\mathbf{q}$ as the generalized coordinates of the system. The Euler-Lagrange equations give for each component $q_{i}$ of $\mathbf{q}$ :

$$
\frac{d}{d t}\left(\frac{\partial L}{\partial \dot{q}_{i}}\right)-\frac{\partial L}{\partial q_{i}}=Q_{i}
$$

where $Q_{i}$ describes the generalized force associated to $q_{i}$ :

$$
Q_{i}=\int_{\mathcal{C}_{\mathbf{q}}} \mathbf{f}^{\top}(u) \frac{\partial \mathcal{C}_{\mathbf{q}}(u)}{\partial q_{i}} d u .
$$

f are the dissipative forces, $L$ the Lagrangian, it is given by $L=T-U$ with $T$ the kinetic energy and $U$ the potential energy. $T$ writes as

$$
T=\int_{\mathcal{C}_{\mathbf{q}}} \frac{1}{2} \mu\left\|\mathcal{C}_{t}\right\|^{2} d u
$$

with $\mathcal{C}_{t}=\partial \mathcal{C}_{\mathbf{q}} / \partial t$ and $\mu$ the mass of each point supposed to constitute $\mathcal{C}_{\mathbf{q}}$. Most often, $U$ is described from the Tikhonov regularization terms:

$$
U=\int_{\mathcal{C}_{\mathbf{q}}} \frac{1}{2} k_{1}\left\|\mathcal{C}_{u}\right\|^{2} d u+\int_{\mathcal{C}_{\mathbf{q}}} \frac{1}{2} k_{2}\left\|\mathcal{C}_{u u}\right\|^{2} d u
$$

where $\mathcal{C}_{u}=\partial C q / \partial u, \mathcal{C}_{u u}=\partial C q / \partial u$. The scalar $k_{1}$ will tend to limit the extension of $\mathcal{C}_{\mathbf{q}}$ while $k_{2}$ will tend to limit its curvature. According to (1), we have

$$
\left\{\begin{array}{l}
\mathcal{C}_{t}=\sum_{l} \dot{q}_{l} \boldsymbol{\Phi}_{l} \\
\mathcal{C}_{u}=\sum_{l} q_{l} \boldsymbol{\Phi}_{l}^{\prime} \quad\left(\text { with } \boldsymbol{\Phi}_{l}^{\prime}=\partial \boldsymbol{\Phi}_{l} / \partial u\right) \\
\mathcal{C}_{u u}=\sum_{l} q_{l} \boldsymbol{\Phi}_{l}^{\prime \prime} \quad\left(\text { with } \boldsymbol{\Phi}_{l}^{\prime \prime}=\partial^{2} \boldsymbol{\Phi}_{l} / \partial^{2} u\right) .
\end{array}\right.
$$

Therefore, it becomes straightforward to evaluate the contribution of $U$ in (2): we have $\partial U / \partial \dot{q}_{i}=0$ and

$$
\frac{\partial U}{\partial q_{i}}=\int_{\mathcal{C}_{\mathbf{q}}}\left(\sum_{l} k_{1} q_{l} \boldsymbol{\Phi}_{l}^{\prime \top} \boldsymbol{\Phi}_{i}^{\prime}+\sum_{l} k_{2} q_{l} \boldsymbol{\Phi}_{l}^{\prime \prime \top} \boldsymbol{\Phi}_{i}^{\prime \prime}\right) d u .
$$

Similarly, we have $\partial T / \partial q_{i}=0$ and

$$
\frac{1}{2} \frac{\partial\left\|\mathcal{C}_{t}\right\|^{2}}{\partial \dot{q}_{i}}=\sum_{l} \dot{q}_{l} \boldsymbol{\Phi}_{l}^{\top} \boldsymbol{\Phi}_{i}
$$

leading to

$$
\frac{d}{d t}\left(\frac{\partial T}{\partial \dot{q}_{i}}\right)=\int_{\mathcal{C}_{\mathbf{q}}} \mu\left(\sum_{l} \ddot{q}_{l} \boldsymbol{\Phi}_{l}^{\top} \boldsymbol{\Phi}_{i}\right) d u .
$$

Thus, from (7) and (9), the left part of (2) can be evaluated. Concerning, the right part, we first consider a force due to a viscous friction $\mathbf{f}_{\text {vis }}=-\gamma \mathcal{C}_{t}$, where $\gamma$ is the viscosity of the medium, and, second, a force $\mathbf{f}_{i m}$ due to the image. In addition, we suppose that $\mathbf{f}_{i m}$ derives from a potential energy term $E_{i m}: \mathbf{f}_{i m}=-\boldsymbol{\nabla} E_{i m}$ where $\boldsymbol{\nabla} E_{i m}$ denotes the spatial gradient of $E_{i m}$ in the image. In this paper, we will use $E_{i m}=-\|\nabla \mathbf{I}\|$. Concerning the contribution of $\mathbf{f}_{v i s}$, we have:

$$
\int_{\mathcal{C}_{\mathbf{q}}} \mathbf{f}_{v i s}^{\top} \frac{\partial \mathcal{C}_{\mathbf{q}}(u)}{\partial q_{i}} d u=-\int_{\mathcal{C}_{\mathbf{q}}} \gamma\left(\sum_{l} \dot{q}_{l} \mathbf{\Phi}_{l}\right)^{\top} \boldsymbol{\Phi}_{i} d u .
$$

Finally, from (7), (9) and (10), (2) can be written under a matrix form:

$$
\mathbf{M} \ddot{\mathbf{q}}+\mathbf{C} \dot{\mathbf{q}}+\mathbf{K q}=\mathbf{Q}(\mathbf{q})=-\int_{\mathcal{C}_{\mathbf{q}}} \nabla E_{i m} \frac{\partial \mathcal{C}_{\mathbf{q}}(u)}{\partial \mathbf{q}} d u
$$

where $\mathbf{M}=\left[M_{m n}\right], \mathbf{C}=\left[C_{m n}\right]$ and $\mathbf{K}=\left[K_{m n}\right]$ with

$$
\left\{\begin{array}{l}
M_{m n}=\mu \int_{\mathcal{C}_{\mathbf{q}}} \boldsymbol{\Phi}_{m}^{\top} \boldsymbol{\Phi}_{n} d u \\
C_{m n}=\gamma \int_{\mathcal{C}_{\mathbf{q}}}^{\boldsymbol{\Phi}_{m}^{\top} \boldsymbol{\Phi}_{n} d u} \\
K_{m n}=k_{1} \int_{\mathcal{C}_{\mathbf{q}}} \boldsymbol{\Phi}_{m}^{\prime \top} \boldsymbol{\Phi}_{n}^{\prime} d u+k_{2} \int_{\mathcal{C}_{\mathbf{q}}} \boldsymbol{\Phi}_{m}^{\prime \prime \top} \boldsymbol{\Phi}_{n}^{\prime \prime} d u .
\end{array}\right.
$$

To solve the differential system (11), it is more convenient to set the mass $\mu$ to zero as proposed in [1]. Moreover, we use an explicit Euler's method, leading to

$$
\mathbf{q}_{k+1}=\mathbf{q}_{k}+\Delta t \mathbf{C}^{-1}(\mathbf{Q}(\mathbf{q})-\mathbf{K q})
$$

where $\Delta t$ is the sampling rate. In practice, its value must be small since $E_{i m}$ is only valid in a small domain around $\mathbf{q}_{k}$.

Lets us point out that the computation of matrices $\mathbf{C}$ and $\mathbf{K}$ can be time consuming. Indeed, they are obtained from an integration along $\mathcal{C}_{\mathbf{q}}$. Besides, the terms to integrate may be themselves complex to compute. That is why we propose in the next section a way to decrease the computation time of (13). It is based on a judicious choice for $\boldsymbol{\Phi}_{l}$.

\section{USING A POLAR DESCRIPTION}

A way to simplify (12) is to use orthogonal functions such that their first and second derivative are also orthogonal in the sense of the inner product defined by the integrals in (12). Note that it cannot be achieved using traditional orthogonal polynomials. Conversely, it can be done using a polar description of the contour $\mathcal{C}_{\mathbf{q}}$ :

$$
\mathcal{C}_{\mathbf{q}}(u): \mathbf{x}(u)=\mathbf{x}_{c}+\rho_{\mathbf{q}}(u)\left(\begin{array}{c}
\cos u \\
\sin u
\end{array}\right)
$$

and by performing a Fourier expansion of the radius $\rho_{\mathbf{q}}(u)$ :

$$
\rho_{\mathbf{q}}(u)=q_{0}+\sum_{k=1}^{k=h} q_{k} \cos k u+q_{k+h} \sin k u
$$


where $h$ is the number of harmonics. Thus, we have $\mathbf{q}=$ $\left(q_{0}, \cdots, q_{2 h}\right)$ and consequently:

$$
\mathbf{\Phi}_{l}=\left(\begin{array}{c}
\cos u \cos l u \\
\sin u \cos l u
\end{array}\right) \quad \text { for } 0 \leq l \leq h
$$

and

$$
\boldsymbol{\Phi}_{l}=\left(\begin{array}{c}
\cos u \sin (l-h) u \\
\sin u \sin (l-h) u
\end{array}\right) \quad \text { for } h<l \leq 2 h .
$$

In that case, it is easy to show that the matrices involved in (12) becomes diagonal and constant. Indeed, we simply have

$$
\begin{gathered}
\mathbf{C}=\gamma \operatorname{diag}(2 \pi, \pi, \cdots, \pi) \\
\mathbf{K}_{1}=k_{1} \operatorname{diag}\left(2 \pi, \pi\left(1+1^{2}\right), \cdots, \pi\left(1+h^{2}\right),\right. \\
\left.\pi\left(1+1^{2}\right), \cdots, \pi\left(1+h^{2}\right)\right) \\
\mathbf{K}_{2}=k_{2} \operatorname{diag}(2 \pi, \\
\pi\left(1+6 \times 1^{2}+1^{4}\right), \cdots, \pi\left(1+6 h^{2}+h^{4}\right), \\
\left.\pi\left(1+6 \times 1^{2}+1^{4}\right), \cdots, \pi\left(1+6 h^{2}+h^{4}\right)\right)
\end{gathered}
$$

with $\mathbf{K}_{1}$ and $\mathbf{K}_{2}$ defined such that $\mathbf{K}=\mathbf{K}_{1}+\mathbf{K}_{2}$.

\section{USING AN AREA-BASED ENERGY TERM}

We are interested in this section to add an external force to the active contour model to help the curve to reach the true contour even if its initialization is far from it. However, adding such a force is time consuming since its contribution to (3) requires the computation of an integral of a complex term. It is the case for example with the pressure forces proposed in [11] where the normal has to be computed at each point. However, as pointed out in [12], this force can also be interpreted as the gradient of an area energy term $E_{\text {area }}$ defined by $E_{\text {area }}=k_{3} S_{\mathbf{q}}$ where $S_{\mathbf{q}}$ denotes the area of the inside region delimited by the curve and $k_{3}$ a scalar. If $k_{3}$ is a positive value, the area will decrease until the contour is reached. Thus, an initial estimation outside the contour is required. Conversely, if we want to consider an initialization inside the contour, a negative value has to be chosen.

Thanks to the polar description, this surface is very simple to compute from the well-known Green-Riemann's formula. Indeed, we have

$$
S_{\mathbf{q}}=\pi\left(q_{0}^{2}+\frac{1}{2} \sum_{k} q_{k}^{2}\right)
$$

that also leads to a very simple way to compute $\partial E_{\text {area }} / \partial q_{i}$ involved in (2). Indeed, we only have to introduce the following diagonal and constant matrix:

$$
\mathbf{K}_{3}=k_{3} \operatorname{diag}(2 \pi, \pi, \cdots, \pi)
$$

that we have to add to the matrices $\mathbf{K}_{1}$ and $\mathbf{K}_{2}$.
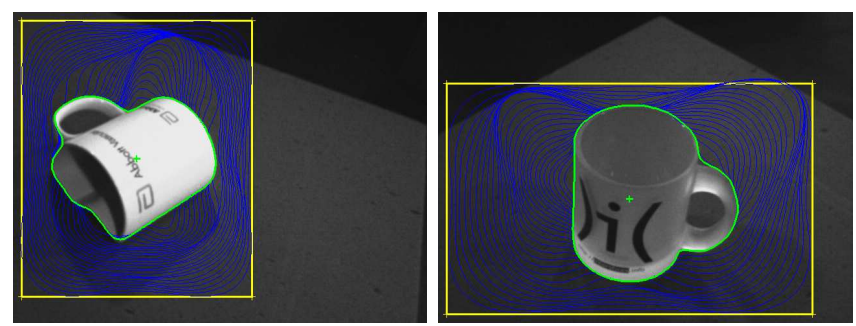

Fig. 1. Robustness in the case of an outside initialization for objects of the everyday life.
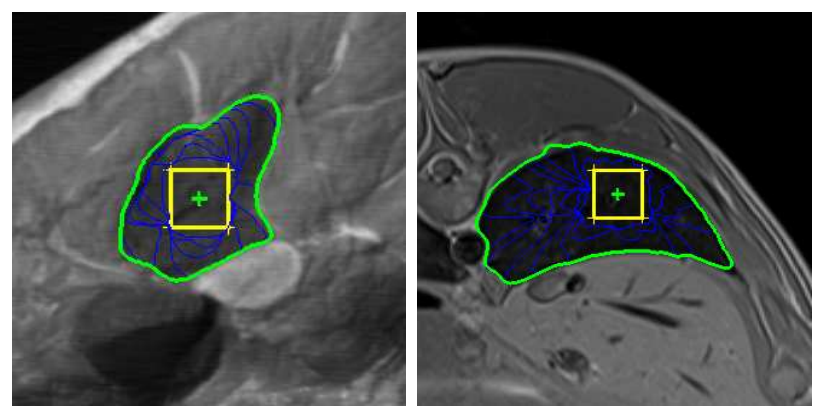

Fig. 2. Robustness in the case of an inside initialization for various objects.

\section{EXPERIMENTAL RESULTS}

The first experiment is devoted to show the robustness of the convergence wrt a bad initialization of the active contour. The final goal of this experiment is to allow disable persons to grasp an object with the help of a vision system and a robot arm mounted on their wheel chair [14]. Therefore, only one click has been used. Fig.1 shows the behavior of the active contour when objects of the everyday life are considered. The yellow quadrilateral is the initial position of the contour, it has been built from the click (the green cross) and the nearest image border. The curves in blue shows the behavior of the active contour during its convergence; the curve in green depicts the active contour when q no more evolves. As can be seen, using a surface-based energy term is very efficient. For this experiment, the following values have been used for both objects: $h=20, \bar{k}_{1}=0, \bar{k}_{2}=5.10^{-4}, \bar{k}_{3}=0.1, \bar{\gamma}=500$ (where $\gamma=\bar{\gamma} \Delta t$ and $k_{i}=\bar{k}_{i} \Delta t$ ).

The second experiment (see Fig.2) is also devoted to the effectiveness of the initialization process. An inside initialization has been considered. Here we have chosen : $h=30$, $\bar{k}_{3}=-0.2$ (the other parameters remain unchanged). The left image shows a segmentation of a red muscle of a raw ham. The right one, the segmentation of MRI image of a pork lung. Here again, despite bad initializations, the contour converges to the true one.

The goal of the last experiment is to show the capability of our approach to achieve real-time applications (see Fig.3). Indeed, we are interested here to control by visual servoing [10] 

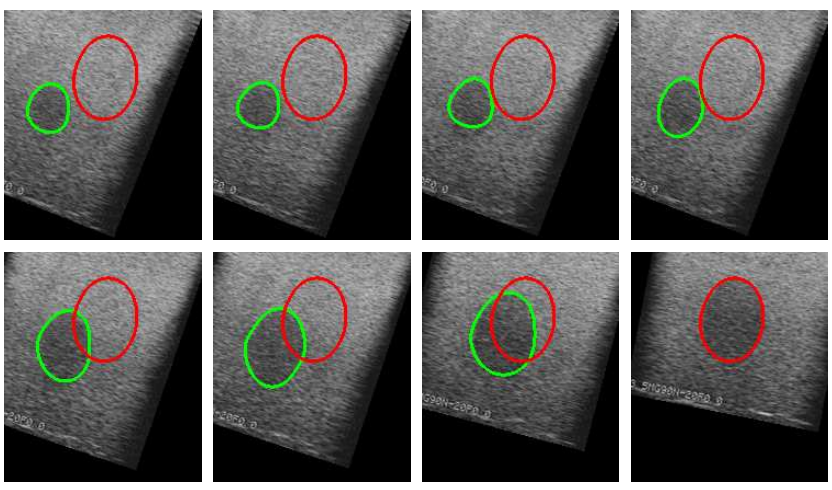

Fig. 3. Tracking contour to achieve a visual servoing task.

the motion of a $2 \mathrm{D}$ ultrasound probe held by a robot. The control law is designed so that the current contour (the green one) reaches a learned contour (the red one) leading to a correct position of the probe. More precisely, the control law is based on some 2D images moments (computed from the vector $\mathbf{q}$, see [15] for more details). Therefore, to achieve such a task the current contour has to be tracked to compute the control law. To do that, $\mathbf{q}_{k-1}$ is used to initialize the contour at time $k$. Using a polar description allowed to reach the video rate. For this experiment, we have used: $h=6, \bar{k}_{1}=0$, $\bar{k}_{2}=1.10^{-2}, \bar{k}_{3}=0$ and $\bar{\gamma}=200$.

\section{CONCLUSION}

We have presented in this paper a physics-based deformable model in the case where the contour is described by a polar description. We have shown that this representation leads to a simple evolution equation of the contour suitable for real-time applications as contour tracking for visual servoing issues. In addition, we have also shown that, with this representation, it is very easy to introduce an additional area-based energy term that efficiently deals with a contour initialization far from the contour to reach. Experiments on various objects have validated our approach as well to perform image segmentation as for contour tracking.

\section{REFERENCES}

[1] D. Terzopoulos and D. Metaxas, "Dynamic 3d models with local and global deformations : deformable superquadrics," IEEE Trans. on Pattern Analysis and Machine Intelligence, vol. 13, pp. 703-713, May 1991.

[2] M. Kass, A. Witkin, and D. Terzopoulos, "Snakes: Active contour models," Int. Journal of Computer Vision, vol. 1, no. 4, pp. 321-331, 1987.

[3] C. Tauber, H. Batatia, G. Morin, and A. Ayache, "Robust b-spline snakes for ultrasound image segmenta- tion," IEEE Computers in Cardiology, vol. 31, pp. 325328, 2004.

[4] V. Caselles, R. Kimmel, and G. Sapiro, "Geodesic active contours," Int. Journal of Computer Vision, vol. 22, no. 1, pp. 61-79, 1997.

[5] P. Brigger, J. Hoeg, and M. Unser, "B-spline snakes: a flexible tool for parametric contour detection," IEEE Trans. on Image Processing, vol. 9, no. 9, pp. 14841496, September 2000.

[6] B. Bascle, P. Bouthemy, N. Deriche, and F. Meyer, "Tracking complex primitives in an image sequence," in IAPR Int. Conf. on Pattern Recognition, Jerusalem, October 1994, pp. 426-431.

[7] F. Precioso, M. Barlaud, T. Blu, and M. Unser, "Robust real-time segmentation of images and videos using a smoothing-spline snake-based algorithm," IEEE Trans. on Image Processing, 2005.

[8] R. Malladi, J.A. Sethian, and B.C. Vemuri, "Shape modeling with front propagation: A level set approach," IEEE Trans. on Pattern Analysis and Machine Intelligence, vol. 17, no. 2, pp. 158-175, 1995.

[9] C. Xu and L. Prince, "Snakes, shapes, and gradient vector flow," IEEE Trans. on Image Processing, vol. 7, pp. 359-369, March 1998.

[10] F. Chaumette and S. Hutchinson, "Visual servo control, Part I: Basic approaches," IEEE Robotics and Automation Magazine, vol. 13, no. 4, pp. 82-90, December 2006.

[11] L.D. Cohen, "On active contour models and balloons," CVGIP: Image Understanding, vol. 53, no. 2, pp. 211218, 1991.

[12] L.D. Cohen and I. Cohen, "Finite-element methods for active contour models and balloons for 2-d and 3-d images," IEEE Trans. on Pattern Analysis and Machine Intelligence, vol. 15, no. 11, pp. 1131-1147, November 1993.

[13] B. Leroy, I. Herlin, and L.D. Cohen, "Multi-resolution algorithms for active contour models," in Int. Conf. Analysis and Optimization of Systems, Paris, France, June 1996, vol. 4, pp. 58-65.

[14] C. Dune, E. Marchand, C. Collewet, and C. Leroux, "Active rough shape estimation of unknown objects," in IEEE/RSJ Int. Conf. on Intelligent Robots and Systems, IROS'08, Nice, France, September 2008, pp. 36223627.

[15] R. Mebarki, A. Krupa, and C. Collewet, "Automatic guidance of an ultrasound probe by visual servoing based on b-mode image moments," in Int. Conf. on Medical Image Computing and Computer Assisted Intervention (MICCAI), New York, USA, September 2008. 